\title{
Estudio de la micocenosis de macromicetos del Parque Natural del Monte Aloia (Pontevedra, España)
}

\author{
por \\ Purificación Lorenzo \& M. ${ }^{a}$ Luisa Castro \\ Laboratorio de Micoloxía, Facultade de Bioloxía, Universidade de Vigo, E-36310 Vigo \\ purilorenzo@uvigo.es; lcastro@uvigo.es
}

\begin{abstract}
Resumen
Lorenzo, P. \& Castro, M.L. 2009. Estudio de la micocenosis de macromicetos del Parque Natural del Monte Aloia (Pontevedra, España). Anales Jard. Bot. Madrid 66S1: 151-156.

En este trabajo se estudia la composición de la comunidad macrofúngica del Parque Natural del Monte Aloia (Tui, Pontevedra), así como su relación con los distintos biotopos del área seleccionada. La distribución de las especies en los micotopos caracterizados se analizó empleando el índice de similitud de Bray \& Curtis (análisis de conglomerado). La similitud y disimilitud promedio entre biotopos se ha determinado mediante los métodos de ordenación de Escalamiento Multidimensional no Métrico (MDS) y de análisis de Similitud Porcentual (SIMPER). Todos fueron analizados utilizando el programa estadístico PRIMER (Plymouth Routines In Multivariate Ecological Research, versión 6 para Windows).
\end{abstract}

Palabras clave: Península Ibérica, Galicia, Monte Aloia, micocenosis, micotopo, macromicetes, índice de similitud, ordenación MDS, análisis SIMPER.

\section{Introducción}

El Parque Natural del Monte Aloia se sitúa dentro del término municipal de Tui, al sur de la provincia de Pontevedra (España), próximo a la desembocadura del río Miño.

Abarca una superficie de 746,29 ha y se asienta sobre un sustrato granítico, de composición ácida, en una región climatológicamente húmeda, como corresponde al noroeste ibérico, en la que los meses de verano pueden ser considerados secos.

Se trata de un parque natural atípico, ya que la vegetación potencial, el robledal atlántico de Quercus robur, ocupa poca extensión y se encuentra en mal estado de conservación. Alrededor de 430 ha están ocupadas por Pinus pinaster y algunas manchas de P. syl-

\begin{abstract}
Lorenzo, P. \& Castro, M.L. 2009. Study of the mycocoenosis of macromycetes in the Parque Natural do Monte Aloia (Pontevedra, Spain). Anales Jard. Bot. Madrid 6651: 151-156 (in Spanish).

We study the composition of macrofungal communities in the Natural Reserve of Monte Aloia (Tui, Pontevedra) and its relation with different biotopes in the selected area. The distribution of species in the selected mycotopes was analyzed using the Bray \& Curtis similarity Index. (Cluster analysis). The mean values of similarity and dissimilarity among biotopes was determined using Multidimensional non-metric Scaling (MDS) and the analysis of Porcentual Similarity (SIMPER). All biotopes were analyzed using the statistical program PRIMER (Plymouth Routines In Multivariate Ecological Research, version 6 for Windows).
\end{abstract}

Keyword: Iberian Peninsula, Galicia, Monte Aloia, mycocoenosis, mycotope, macromycetes, similarity index, MDS distribution, SIMPER analysis.

vestris y $P$. radiata. También se observan otras plantaciones, más o menos extensas, con especies invasoras y de interés forestal como Robinia pseudoacacia, Acacia dealbata, A. melanoxylon y Eucalyptus globulus, junto con varios rodales de coníferas de gran porte como Chamaecyparis lawsoniana o Cryptomeria japonica. Sólo en el margen de pequeños regatos se pueden ver restos discontinuos de la ripisilva autóctona, relativamente bien conservada y constituida esencialmente por Alnus glutinosa, Corylus avellana y Betula alba.

Este trabajo está integrado en el proyecto PGIDT 99MA30101 (Xunta de Galicia), relativo al estudio de la micobiota de los Parques Naturales de la Franja Atlántica. Este conocimiento es imprescindible de 


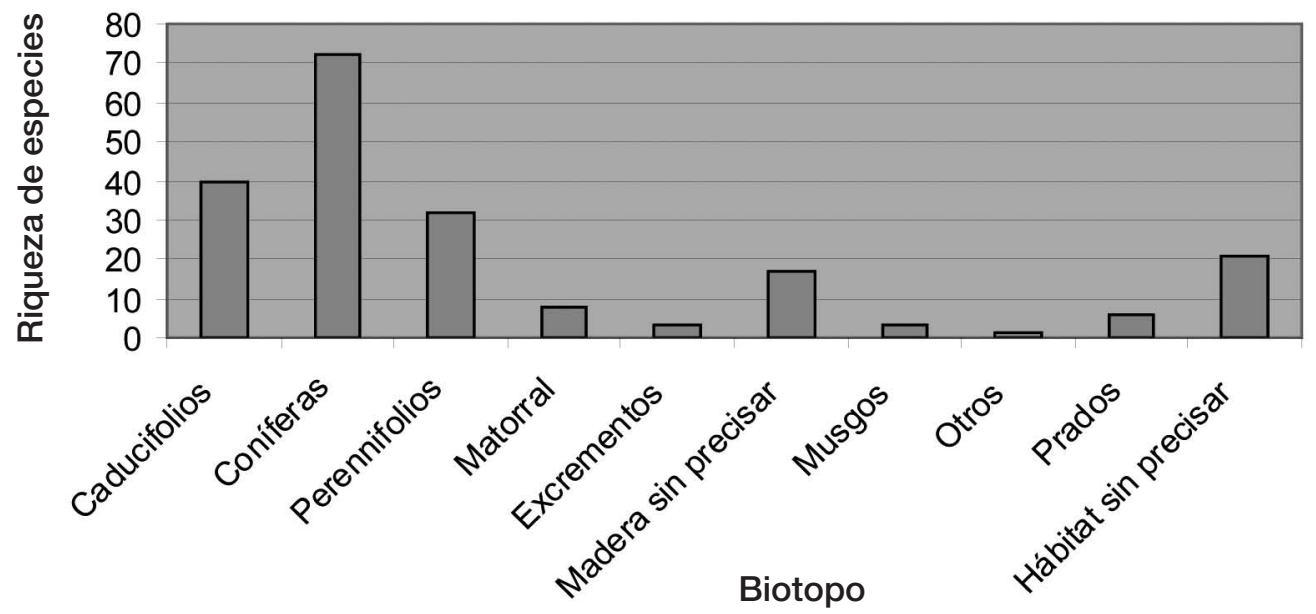

Fig. 1. Riqueza específica en función del biotopo asociado.

cara a los futuros proyectos relacionados tanto con la protección y conservación de especies (Hawksworth, 1991) como con la gestión forestal sostenida (Palm \& Chapela, 1997) dentro y fuera de las áreas protegidas, del mismo modo que se está haciendo en el resto de Europa, con el fin de evitar que la sobreexplotación conduzca a un declive claro de la biodiversidad (Shiva \& Verma, 2002), especialmente de especies micorrícicas (Arnolds, 1991), grupo en el que se incluyen la mayoría de las especies comestibles y comercializables del noroeste Ibérico.

\section{Material y métodos}

Para determinar la composición de la comunidad fúngica y su relación con los distintos biotopos del Parque Natural, se realizaron un total de 34 visitas al área de estudio, entre noviembre de 1999 y septiembre de 2003.

La riqueza macrofúngica se ha obtenido a partir del sumatorio de especies (riqueza específica) o, en su caso, de géneros (riqueza genérica) recolectados e identificados en el área de estudio.

Se elaboraron tablas similares en función de las formas de vida (saprotróficos, micorrícicos y parásitos) o del sustrato al que se asocia, en base tanto a las observaciones directas en el campo como a los datos existentes en la bibliografía consultada (Heiden \& Sanders, 1997; Lee \& Lee, 2004).

Para el tratamiento de los datos, los micotopos prospectados se han subdivido en 10 grupos, a los que denominamos: coníferas, caducifolios, perennifolios, madera sin precisar, prados, matorrales, musgos, excrementos, otros y hábitat sin precisar. En cada visita, las muestras fueron tomadas al azar aplicando la misma unidad de esfuerzo en todos ellos.
El biotopo denominado coníferas está representado por las especies recolectadas bajo Pinus, sobre todo $P$. pinaster, dominante en el Parque Natural, así como otras coníferas ornamentales y/o de interés forestal, con gran porte y alto grado de cobertura, como Chamaecyparis lawsoniana, Pseudotsuga menziesii o Cryptomeria japonica.

En el de árboles caducifolios se incluyen las recolectadas en masas autóctonas, discontinuas y bastante degradadas, de Quercus robur, así como bajo ejemplares aislados o en rodales de Castanea (C. sativa, C. crenata, ...) y Robinia pseudoacacia; además de otras especies propias de ripisilva como Betula alba, Corylus avellana y Alnus glutinosa.

El de árboles perennifolios está representado por las recolectadas principalmente bajo Eucalyptus globulus, así como diversas especies de Acacia (A. dealbata, $A$. decurrens, $A$. melanoxylon, ...), normalmente plantadas formando rodales. La micobiota asociada a estas formaciones alóctonas manifiesta cierto interés, que se refleja en la aparición de los táxones asociados a ellas, como Laccaria lateritia o Descolea maculata, relacionados preferentemente con eucaliptos.

Todas las muestras recogidas sobre madera en descomposición o de las que, por su estructura, nos era imposible identificar la especie vegetal se incluyeron dentro del grupo 'madera sin precisar'.

Debido a las características ecológicas que generan los musgos, especialmente los situados en los taludes con orientación norte, con un alto grado de humedad, diferenciamos la micobiota que crece asociada exclusivamente a estas formaciones, como es el caso de $C y$ phellostereum laeve.

También, en el ámbito del Parque Natural se observan zonas de prados de diente y campos baldíos, ricos 
en nitrógeno y elevada insolación, que únicamente presentan vegetación herbácea, lo que hemos denominado prados.

Se han incluido en el grupo 'matorral' las zonas carentes de vegetación arbórea, con suelos especialmente degradados, junto con las vías de arena y tierra compactada, que sirven de comunicación en el interior del parque, con taludes laterales y escasa influencia de la vegetación circundante, a excepción de pequeños brotes de Cistus sp., Acacia melanoxylon, Eucalyptus globulus o Pinus pinaster, que no llegan a sobrevivir ni a formar densos matorrales o bosquetes, aunque sí pueden llevar asociadas algunas especies micorrizógenas, casi siempre con carpóforos de muy pequeño tamaño.

Dentro de las lindes del Parque Natural, se crían caballos y vacas, que pastan durante todo el año en la zona, generando una micoflora coprófila asociada.

Se incluyen otros dos grupos, uno definido como 'otros', que reúne las especies encontradas en hábitats minoritarios y no se corresponde con ninguno de los anteriores, y el de 'hábitat sin precisar', para aquel material de herbario del que no hemos podido conocer con exactitud su biotopo.

$\mathrm{El}$ análisis de datos se ha elaborado a partir de una matriz de presencia/ausencia para cada biotopo. Para analizar la distribución de los macromicetos entre los biotopos caracterizados se realizó un análisis de conglomerado, empleando el índice de similitud de Bray \& Curtis (1957).

Los resultados se representan en un dendrograma que, para determinar la similitud y disimilitud promedio entre biotopos, se ha confrontado con el método de ordenación de escalamiento multidimensional no

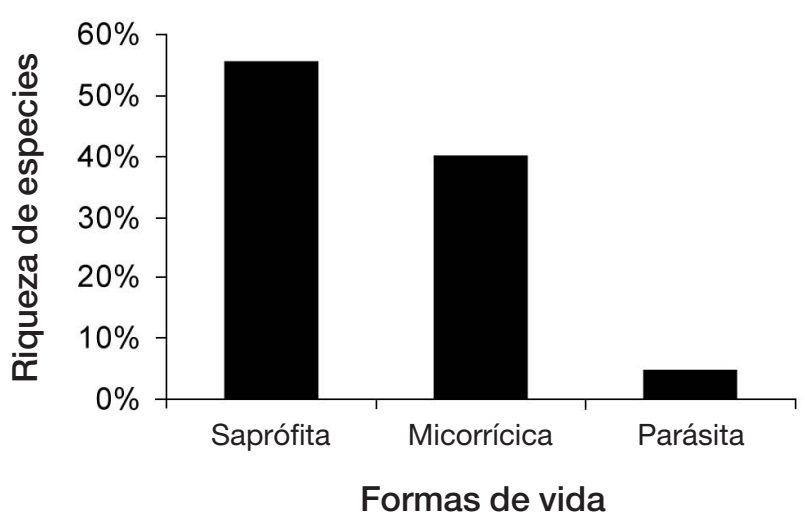

Fig. 2. Distribución de especies según su forma de vida. métrico (Multidimensional Scaling, MDS) y el de Análisis de Similitud Porcentual (Similarity Percentages, SIMPER), logrando de esta manera observar gráficamente la distribución de la micoflora existente. Todos los análisis fueron realizados utilizando el programa estadístico PRIMER (Plymouth Routines In Multivariante Ecological Research, versión 6 para Windows).

\section{Resultados y discusión}

La riqueza macrofúngica estudiada para el Parque Natural del Monte Aloia está incluida en 9 órdenes, 41 familias, 77 géneros y 165 especies, siguiendo la clasificación de Kirk \& al. (2001); el catálogo ordenado por hábitats está pendiente de ser publicado (Lorenzo \& Castro, 2009).

Analizando los biotopos reconocidos en el Parque Natural, el más importante en cuanto a riqueza específica corresponde, como cabría esperar por la extensión que ocupa, con el bosque de coníferas, donde se recogieron 72 especies. Las otras unidades arbóreas, con caducifolios o perennifolios, le siguen, en cuanto a riqueza macrofúngica, con 40 y 32 especies, respectivamente.

El espectro de formas de vida está dominado por especies saprotróficas, con 91 táxones, lo que representa un $55 \%$ del total de especies encontradas, seguido por las especies biotróficas o micorrícias con 66 especies, que representan un $40 \%$ del total (Fig. 2).

Sólo se han encontrado ocho especies potencialmente patógenas, un $5 \%$ del total, lo que pone de manifiesto, inicialmente, el buen estado fitosanitario de las matas arbóreas del Parque Natural, lo que no sorprende ya que desde mediados del siglo pasado ha sido forestado y cuidado por los ingenieros del Centro

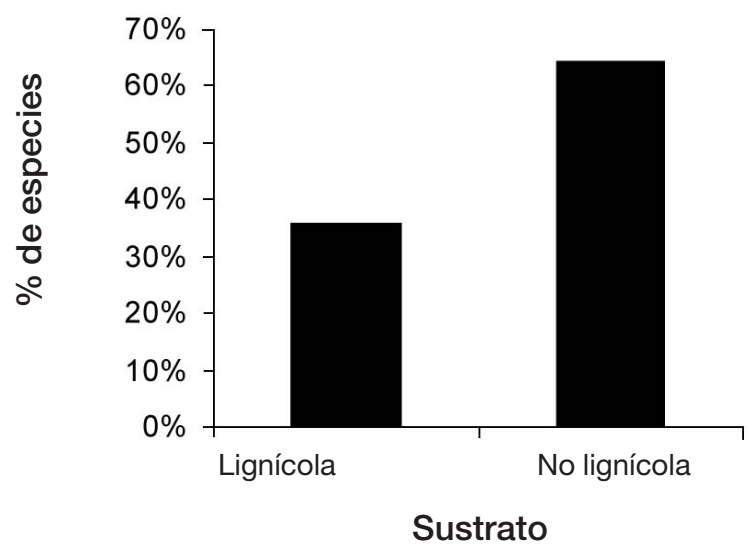

Fig. 3. Distribución de especies según el sustrato sobre el que se desarrollan. 
Forestal de Lourizán y el Vivero de Areas (Tui). El vigor de los árboles es satisfactorio y de acuerdo con lo indicado por por Ortega \& al. (2002) y Ortega \& Navarro (2004), para Andalucía, el papel de dichos táxones patógenos está claramente limitado.

Entre las recolecciones, se observa un elevado porcentaje de especies no lignícolas (64\% del total). Bajo esta denominación se agrupan especes micorrícicas y saprotróficas recogidas directamente en el suelo. El número de especies lignícolas es menor $36 \%$ del total) y engloba tanto especies recolectadas sobre árboles vivos como sobre ramas caídas o tocones.

En el dendrograma representado en la Fig. 4, se muestra la separación de los grupos (biotopos), de acuerdo con el índice de similitud de la flora macrofúngica presente para cada uno de ellos, en tres grupos.

El valor promedio de similitud obtenido con el análisis de estrategia de agrupación (SIMPER) para el primer grupo, asociado a comunidades no arboladas, de vegetación baja, fue de un 17,0 \%. Para el segundo, asociado a comunidades de bosques, resultó un 25,0 \% , siendo la disimilitud media entre ambos grupos de un $89,0 \%$.

En el tercer grupo, asociado a especies coprófilas, la similitud no se puede calcular al tener tres muestras y, por ello, presenta un valor promedio de disimilitud del $97,6 \%$ y $96,1 \%$ respecto al primer y segundo grupos, respectivamente. Además, esta figura permite también observar la disimilitud existente dentro de cada grupo.

Los agrupamientos ilustrados en el dendrograma (Fig. 4) coinciden también con la Fig. 5, que refleja la ordenación (MDS) con un valor de estrés de 0,05, dato que se relaciona con una buena ordenación.

Por otra parte, como la fructificación fúngica depende de las características propias de la especie, de la climatología de la zona y del entorno, es fácil deducir que uno de los principales factores que influye sobre este desarrollo es la vegetación (Winterhoff, 1992). Sin embargo, la afinidad de los diversos macromicetos por una determinada especie vegetal es muy variable ya que, en general, viene dada tanto por la especifidad del hongo por el sustrato como por el propio tipo de nutrición del mismo.

Los tipos de agrupamientos obtenidos mediante la ordenación (MDS) muestran una mayor similitud entre las especies de gran porte, que proporcionan amplia cobertura vegetal, es decir, los árboles (G2. bosques), seguida de las especies de menor tamaño y co-

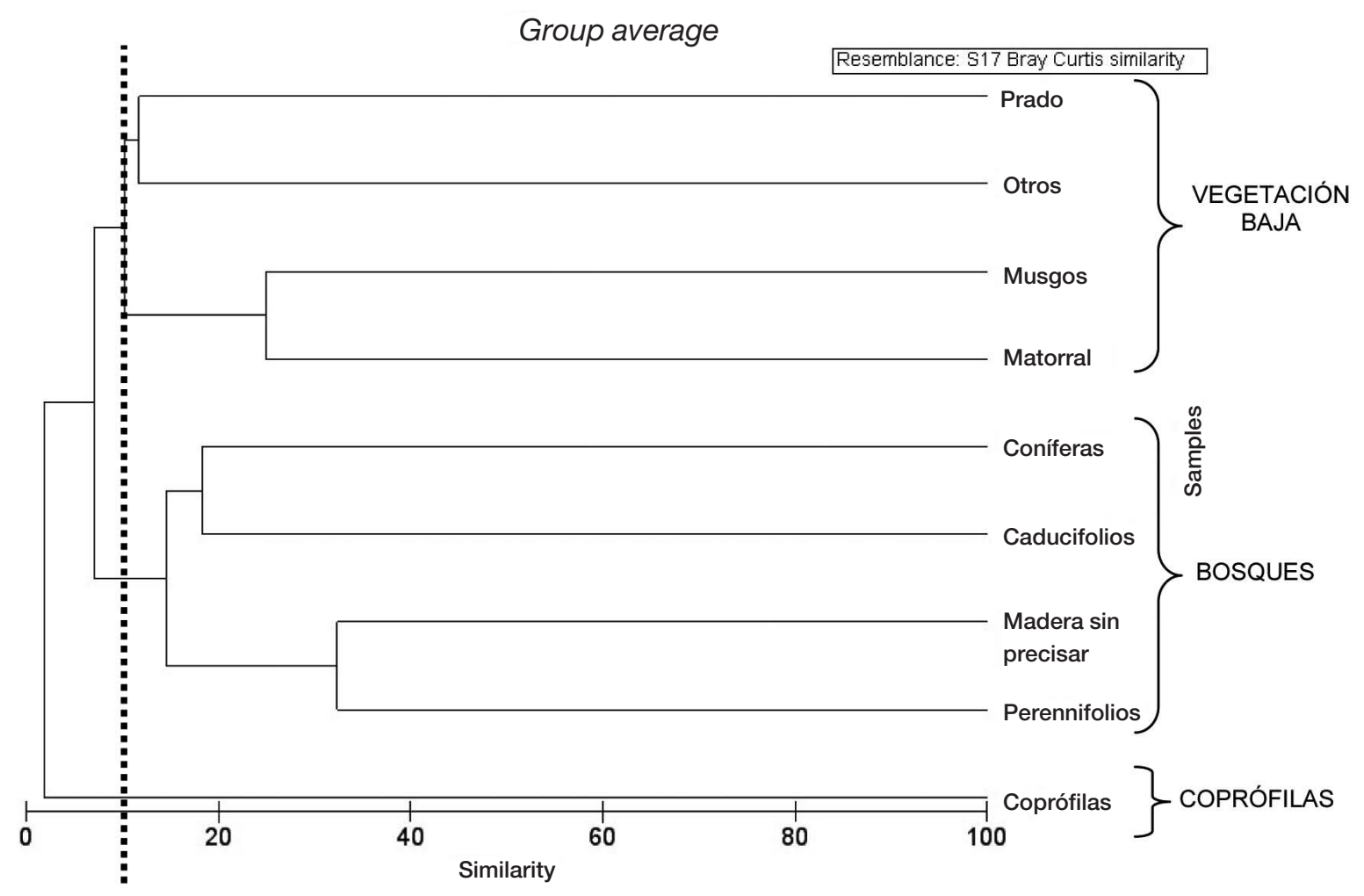

Fig. 4. Dendrograma basado en las especies encontradas en los diferentes biotopos. 


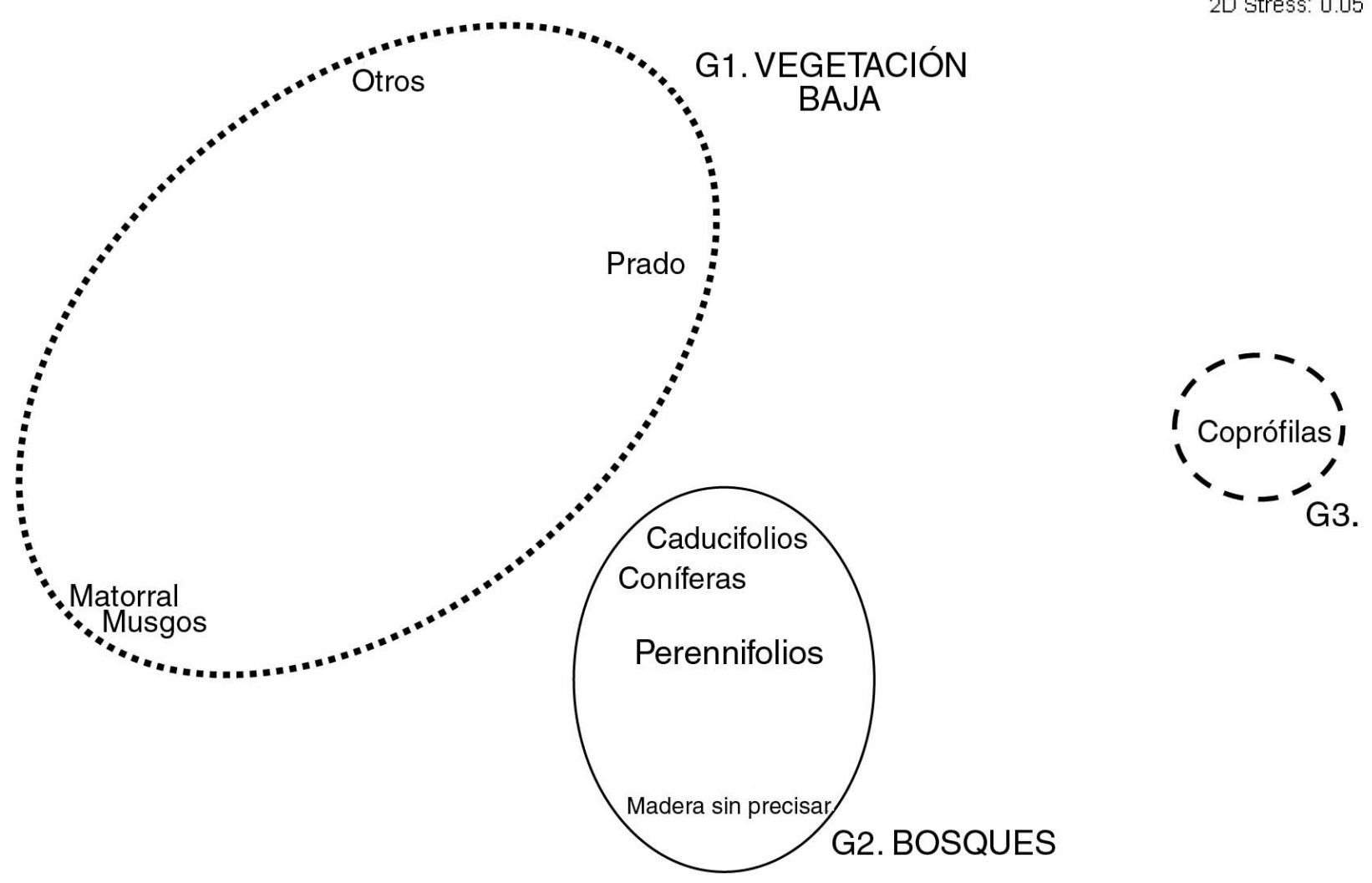

Fig. 5. Gráfico de ordenación (MDS) basado en las especies encontradas en los diferentes biotopos.

bertura, herbáceas o matorral bajo (G1. vegetación baja).

En la Tabla 1 se muestran las especies con mayor representatividad numérica, superior al 50\%, en cada uno de los grupos definidos según los criterios anteriores. En el primer grupo, tan sólo cinco especies fueron mayoritariamente responsables de la similaridad dentro de ese grupo, mientras que en el segundo aparecen once especies. El tercer grupo sólo incluye especies coprófilas, por lo que no se establecen estas comparativas.

Los táxones que contribuyen a esta similaridad corresponden a especies generalistas, sin requerimientos específicos y/o asociaciones a hábitats concretos. Especies como Laccaria lateritia o Paxillus involutus, que aparecen en los dos tipos de agrupamiento, indican poca especificidad de ambas por la vegetación circundante.

Otro de los factores que condicionan la fructificación de los macromicetos es el clima, en especial temperatura, humedad y luz, ya que los macromicetos poseen un intervalo óptimo de crecimiento y fructifica-
Tabla 1. Especies responsables de la similaridad dentro de cada grupo.

\begin{tabular}{|l|l|}
\hline G1. Vegetación baja & \multicolumn{1}{|c|}{ Especies } \\
& $\begin{array}{l}\text { Hygrophoropsis aurantiaca } \\
\text { Laccaria lateritia } \\
\text { Paxillus involutus } \\
\text { Scleroderma citrinum } \\
\text { Suillus bovinus }\end{array}$ \\
\hline G2. Bosques & $\begin{array}{l}\text { Amanita rubescens } \\
\text { Astraeus hygrometricus } \\
\text { Bjerkandera adusta } \\
\text { Byssomerulius corium } \\
\text { Ganoderma australe } \\
\text { Laccaria lateritia } \\
\text { Mycena galericulata } \\
\text { Paxillus involutus } \\
\text { Russula cyanoxantha } \\
\text { Russula ochroleuca }\end{array}$ \\
\hline
\end{tabular}




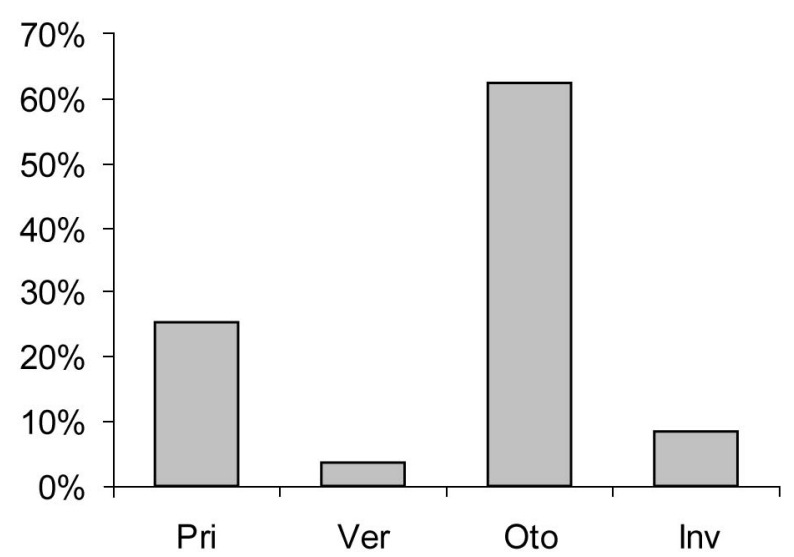

Fig. 6. Distribución de la micetación estacional elaborada a partir de la totalidad del catálogo.

ción, de ahí que la producción de carpóforos no sea homogénea a lo largo del año.

Como puede observarse en la Fig. 6, elaborada a partir del número total de especies recolectadas en el Parque Natural durante el período de estudio, la fructificación se localiza preferentemente en otoño, estación que lleva asociada humedad alta y temperaturas moderadas; mientras que la escasez invernal y estival se relaciona, respectivamente, con bajas temperaturas y sequedad.

\section{Agradecimientos}

Agradecemos a la Xunta de Galicia la concesión del proyecto PGIDT99MA30101, que nos ha permitido desarrollar el trabajo; a la Junta Gestora y a todo el personal del Parque Natural, las facilidades dadas para muestrear las zonas seleccionadas; a Alfredo Justo, la colaboración en la recolecta del material, y a la Dra. Maruxa Álvarez, su ayuda en el campo de la ecología, los programas informáticos y la interpretación de los resultados.

\section{Referencias bibliográficas}

Arnolds, E. 1991. Decline of ectomycorrhizal fungi in Europe. Agr. Ecosyst. Environ. 35: 209-244.

Bray, J.R. \& Curtis, J.T. 1957. An ordination of the upland forest communities of Southern Wisconsin. Ecol. Monogr. 27: 325 349.

Lorenzo, P. \& Castro, L. 2009. Micetación do Parque Natural «Monte Aloia»: diversidade e autoecoloxía. Mykes 12: 1-10 [en prensa].

Hawksworth, D.L. 1991. The fungal dimension of biodiversity: magnitude, significance and conservation. Mycol. Res. 95(6): 641-655.

Heiden, M.A.G. van der \& Sanders, I.R. (eds.). 1997. Mycorrhizal Ecology. Ecol. Stud. 157: 1-469.

Kirk, P.M., Cannon, P.F., David, J.C. \& Stalpers, J.A. (eds.). 2001. Ainsworth et Bisby's Dictionary of the Fungi. $9^{\text {th }}$ Edition. CAB International. Wallingford (UK).

Lee, K.L. \& Lee, H. 2004. Fungal diversity and fruit body production in relation to vegetation structure, topography and soil properties in a Quercus mongolica forest in central Korea. In: Cripps, C.L. (ed.), Fungi in forest ecosistems: Systematics, diversity and ecology: 267-274. New York Botanical Garden (USA).

Ortega, A. \& Navarro, F.B. 2004. A myco-ecological analysis (lignicolous, Aphyllophorales sensu lato, Basidiomycota) of the Abies pinsapo, Quercus and Pinus forests of Andalusia (Southern Spain). Nova Hedwigia 78: 485-499.

Ortega, A., Esteve-Raventós, F. \& Moreno, G. 2002. Biodiversidad mico-florística del área de distribución del Abies pinsapo en España: aspectos micoecológicos. Cryptogamie mycol. 23(1): 51-69.

Palm, M.E. \& Chapela, I.H. (eds.). 1997. Mycolgy in sustainable development: Expandin concepts, vainishing bordel. Parkway Publishers, Inc. Boone (USA).

Shiva, M.P. \& Verma, S.K. (eds.). 2002. Approaches to sustainable forest management and biodiversity conservation. International Books Distributors. Uttaranchal (India).

Winterhoff, W. (ed.). 1992. Fungi in vegetation science. Kluwer Academic Publishers. Dordrecht (The Netherlands).

Recibido: 26-III-2009

Aceptado: 2-IV-2009 